\title{
Euglenophyceae de ambientes lênticos na planície costeira do Rio Grande do Sul, Sul do Brasil: gêneros Euglena Ehr. e Lepocinclis Perty
}

\author{
Sandra Maria Alves-da-Silva ${ }^{1,2}$ e Jaqueline Rizzi Fortuna ${ }^{1}$
}

Recebido em 11/01/2005. Aceito em 27/09/2005

\begin{abstract}
RESUMO - (Euglenophyceae de ambientes lênticos na planície costeira do Rio Grande do Sul, Sul do Brasil: gêneros Euglena Ehr. e Lepocinclis Perty). São apresentados 28 táxons específicos e infra-específicos dos gêneros Euglena Ehr. e Lepocinclis Perty como resultado do estudo do fitoplâncton e perifíton em ambientes lênticos (lagoas, açude e banhados) da Lagoa do Casamento e ecossistemas associados $\left(30^{\circ} 03^{\prime}-30^{\circ} 34^{\prime} \mathrm{S}\right.$ e $\left.50^{\circ} 25^{\prime}-50^{\circ} 47^{\prime} \mathrm{W}\right)$ e ecossistemas próximos ao Butiazal de Tapes $\left(30^{\circ} 23^{\prime}-30^{\circ} 38^{\prime} \mathrm{S}\right.$ e $\left.51^{\circ} 16^{\prime}-51^{\circ} 29^{\prime} \mathrm{W}\right)$ na planície costeira do Rio Grande do Sul. As coletas abrangeram as estações de outono e primavera de 2003. As áreas úmidas (banhados) associadas à Lagoa do Casamento na primavera de 2003 apresentaram maior riqueza específica destes dois gêneros. Lepocinclis salina Fritsch var. salina foi a espécie que se distinguiu quanto a distribuição por ter ocorrido em $41,2 \%$ do total de amostras analisadas. São novos registros para o estado do Rio Grande do Sul e país, L. playfairiana Defl. var. playfairiana e L. boseensis Xie, Qiu \& Ling.
\end{abstract}

Palavras-chave: Taxonomia, Euglenophyceae pigmentadas, Euglena e Lepocinclis, Brasil

\begin{abstract}
Euglenophyceae of lentic environments at the coastal plain of Rio Grande do Sul State, South of Brazil: genera Euglena Ehr. and Lepocinclis Perty). Twenty eight specific and infra-specific taxa of the genera Euglena Ehr. and Lepocinclis Perty are presented as a result of phytoplankton and periphyton study in lentic systems next to Lagoa do Casamento (30 $03^{\prime}-30^{\circ} 34^{\prime} \mathrm{S}$ and $\left.50^{\circ} 25^{\prime}-50^{\circ} 47^{\prime} \mathrm{W}\right)$ and Butiazal de Tapes ( $30^{\circ} 23^{\prime}-30^{\circ} 38^{\prime}$ S and $51^{\circ} 16^{\prime}-51^{\circ} 29^{\prime} \mathrm{W}$ ), coastal zone of Rio Grande do Sul State. Samples were collected in autumn and spring seasons in 2003. The swamps associated to Lagoa do Casamento in spring of 2003 presented more specific richness of these two genera. Lepocinclis salina Fritsch var. salina distinguished by its distribution, occuring in $41,2 \%$ of the analized samples. Two taxa are new records for the State and Brazil: L. playfairiana Defl. var. playfairiana and L. boseensis Xie, Qiu \& Ling.
\end{abstract}

Key words: Taxonomy, pigmented Euglenophyceae, Euglena and Lepocinclis, Brazil

\section{Introdução}

Este estudo fez parte de um subprojeto dentro do Projeto de Conservação e Utilização Sustentável da Diversidade Biológica Brasileira (PROBIO), trabalho que está sendo desenvolvido por pesquisadores da Fundação Zoobotânica do Rio Grande do Sul desde 2003, cujo objetivo é realizar o levantamento da biodiversidade de algas, plantas, invertebrados e vertebrados para definir estratégias e prioridades de conservação de ecossistemas terrestres e aquáticos da zona costeira do Rio Grande do Sul.

Os locais estudados são ambientes próximos ou em conexão direta com a porção norte da Laguna dos Patos, formando um complexo de remanescentes naturais com representação de diferentes tipos de ecossistemas típicos da zona costeira: as dunas lagunares interiores, os campos litorâneos, os banhados, as matas de restinga e os butiazais.

Foram estudadas duas áreas, a Lagoa do Casamento e seus ecossistemas associados (margem leste da Laguna dos Patos) com uma área de $31.181,013 \mathrm{~km}^{2}$, compreendida entre os municípios de Viamão e Palmares de Sul, localizado a $30^{\circ} 03^{\prime}-30^{\circ} 34^{\prime} \mathrm{S}$ e $50^{\circ} 25^{\prime}-50^{\circ} 47^{\prime} \mathrm{W}$ e os ecossistemas próximos ao Butiazal de Tapes (margem oeste da Laguna dos Patos), com área de $15.103,259 \mathrm{~km}^{2}$, compreendida entre os municípios de Barra do Ribeiro e Tapes a $30^{\circ} 23^{\prime}-30^{\circ} 38^{\prime}$ S e $51^{\circ} 16^{\prime}-51^{\circ} 29^{\prime} \mathrm{W}$ ( Fig. 1).

O clima do Rio Grande do Sul está inserido conforme classificação de Köppen, no tipo Cfa, subtropical úmido, isto é, temperatura do mês mais frio entre $0^{\circ}$ e $18^{\circ} \mathrm{C}(\mathrm{C})$, com precipitação bem distribuída durante o ano e totais superiores a $1.200 \mathrm{~mm}$ (f), mês mais quente com temperaturas superiores a $22^{\circ} \mathrm{C}$ (a).

\footnotetext{
1 Museu de Ciências Naturais da Fundação Zoobotânica do Rio Grande do Sul, Seção de Botânica de Criptógamas, C. Postal 1188, CEP 90001-970, Porto Alegre, RS1, Brasil

2 Autor para correspondência: alvesdasilva@fzb.rs.gov.br, salvesilva@yahoo.com.br
} 
A maior pressão antrópica sobre as áreas naturais remanescentes nessas áreas provém da orizicultura, com presença de monoculturas extensivas que implicaram na destruição de banhados e matas de restinga.

A planície costeira do Rio Grande do Sul apresenta carência no estudo das comunidades fitoplanctônicas que englobem as Euglenophyta, tendo em vista que apenas Alves-da-Silva (1988) publicou um trabalho taxonômico envolvendo 39 táxons específicos e infraespecíficos destas algas na Estação Ecológica do Taim, na porção sul do Estado.

O levantamento de Euglenophyta na planície costeira do Rio Grande do Sul mostrou alta diversidade deste grupo de algas, o que resultará na publicação de vários trabalhos. A presente publicação abrangerá os gêneros Euglena Ehr. e Lepocinclis Perty, o que ampliará o conhecimento e a distribuição geográfica destes dois gêneros de Euglenophyceae pigmentadas para a área em questão.

\section{Material e métodos}

As duas áreas estudadas foram a Lagoa do Casamento e Butiazal de Tapes, assim como, ecossistemas próximos a estas áreas, situadas na planície costeira do Estado do Rio Grande do Sul (Fig. 1). Nestas áreas foram realizadas amostragens de frasco, rede e espremido de macrófitas aquáticas nas margens e centro de vários subsistemas. No presente estudo foram consideradas apenas as coletas marginais realizadas com rede e espremido de macrófitas aquáticas existentes nos diferentes ambientes, no período de outono/2003 (maio e junho) e na primavera/2003 (outubro a dezembro). $\mathrm{Na}$ lagoa do Casamento, as amostragens foram realizadas em várias subáreas, sendo feitas coletas nas estações denominadas de A (lagoa do Casamento e lagoa do Capivari), B (lagoa dos Gateados sul), D (lagoa dos Gateados norte) e G (Pontal do Anastácio e canal Sangradouro), enquanto nos ecossistemas próximos ao Butiazal de Tapes as estações de coleta amostradas foram nas subáreas denominadas localmente de A (englobando a lagoa das Capivaras, banhado do Sphagnum, lagoinha entre dunas) e B (lagoa do Charutão, açude fazenda São Miguel e Lagoa Redonda) que incluíram biótopos aquáticos como lagoas, alagados, açude, canal e banhados (= áreas úmidas) (Tab. 1).

As amostras foram obtidas através de 25 a 30 passagens de rede de plâncton de $25 \mu \mathrm{m}$ de abertura de malha, na porção superficial da água, entre 10 e $15 \mathrm{~cm}$, e realizado espremido de macrófitas aquáticas existentes nos diferentes biótopos.

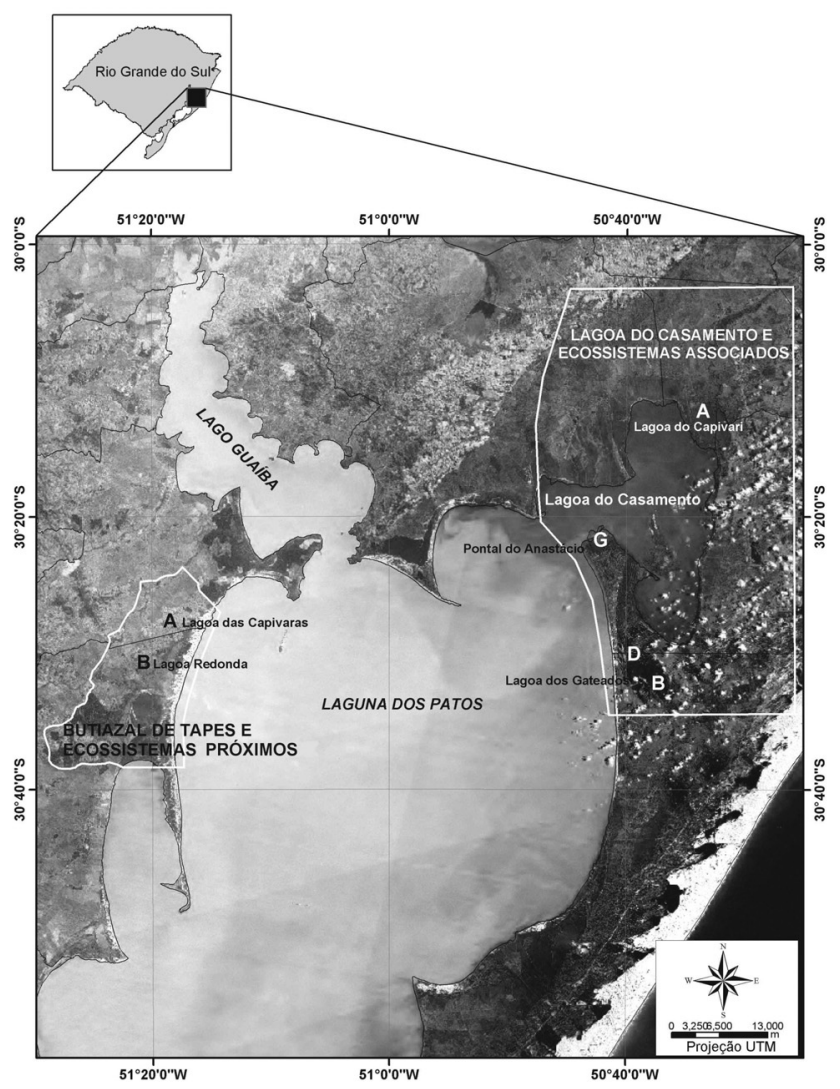

Figura 1. Localização da Lagoa do Casamento e Butiazal de Tapes e seus ecossistemas próximos, com indicação das subáreas estudadas (A, B, D, G), na planície costeira do Rio Grande do Sul, Sul do Brasil.

As amostras coletadas com rede foram subdivididas em duas, uma foi imediatamente fixada e preservada com solução de formaldeído a 3\%, ainda no campo, e a outra foi mantida viva e sob refrigeração durante sete a quinze dias, para observação de certas características morfológicas indispensáveis à identificação taxonômica das euglenofíceas, tais como: metabolia, forma e número de cloroplastos por célula e número e disposição dos grãos de paramido no protoplasto. Algumas amostras perifíticas (espremido de macrófitas aquáticas) foram ainda fixadas com solução de Transeau para serem realizadas futuras comparações com as amostras fixadas com formaldeído. A determinação de algumas espécies foi dificultada uma vez que estas foram encontradas somente em material fixado.

São apresentadas dimensões, relação entre o comprimento e a largura celular (Rc/l), ilustrações, distribuição geográfica mundial, comentários de alguns táxons e descrições somente daqueles ainda não citados anteriormente para planície costeira do Rio Grande do Sul. 
Tabela 1. Lista dos táxons, número das figuras correspondentes, tipo de coleta (E - espremido, $\mathrm{R}$ - rede ), ambientes amostrados (A - alagado, B - banhado, C - canal, L - lagoa) e estação do ano (O - outono/2003, P - primavera/2003).

\begin{tabular}{|c|c|c|c|c|}
\hline Táxons & $\mathrm{N}^{\circ}$ da figura & Tipo de coleta & Ambientes & Estação do ano \\
\hline Euglena acus Ehr. var. acus & 2 & $\mathrm{E}, \mathrm{R}$ & $\mathrm{A}, \mathrm{B}, \mathrm{C}, \mathrm{L}$ & $\mathrm{O} / \mathrm{P}$ \\
\hline E. acus Ehr. var. longissima Defl. & 3 & $\mathrm{E}$ & $\mathrm{B}$ & $\mathrm{O}$ \\
\hline E. agilis H.J. Carter var. agilis & $4-5$ & $\mathrm{E}, \mathrm{R}$ & $\mathrm{B}, \mathrm{C}, \mathrm{L}$ & $\mathrm{O} / \mathrm{P}$ \\
\hline E. allorgei Defl. var. allorgei & 6 & $\mathrm{E}, \mathrm{R}$ & $\mathrm{B}, \mathrm{L}$ & $\mathrm{O} / \mathrm{P}$ \\
\hline E. caudata Hübner var. caudata & 7 & $\mathrm{E}, \mathrm{R}$ & $\mathrm{C}, \mathrm{L}$ & $\mathrm{O} / \mathrm{P}$ \\
\hline E. deses Ehr. var. intermedia Klebs & 8 & $\mathrm{E}, \mathrm{R}$ & $\mathrm{B}, \mathrm{L}$ & $\mathrm{O} / \mathrm{P}$ \\
\hline E. ehrenbergii Klebs var. ehrenbergii & 9 & $\mathrm{E}, \mathrm{R}$ & $\mathrm{B}, \mathrm{L}$ & $\mathrm{O} / \mathrm{P}$ \\
\hline E. limnophila Lemm. var. limnophila & 10 & $\mathrm{E}, \mathrm{R}$ & $\mathrm{B}, \mathrm{C}$ & $\mathrm{O} / \mathrm{P}$ \\
\hline E. limnophila Lemm. var. minor Drez. & 11 & $\mathrm{E}$ & B & $\mathrm{O}$ \\
\hline E. mutabilis Schmitz var. mutabilis & 12 & $\mathrm{E}, \mathrm{R}$ & $\mathrm{B}, \mathrm{L}$ & $\mathrm{O} / \mathrm{P}$ \\
\hline E. oxyuris Schmarda var. oxyuris & 13 & $\mathrm{E}, \mathrm{R}$ & $\mathrm{A}, \mathrm{B}, \mathrm{L}$ & $\mathrm{O} / \mathrm{P}$ \\
\hline E. polymorpha Dang. var. polymorpha & $14-15$ & $\mathrm{R}$ & $\mathrm{C}$ & $\mathrm{P}$ \\
\hline E. sanguinea Ehr. var. sanguinea & $16-17$ & $\mathrm{E}, \mathrm{R}$ & $\mathrm{A}, \mathrm{B}$ & $\mathrm{O} / \mathrm{P}$ \\
\hline E. spirogyra Ehr. var. spirogyra & 18 & $\mathrm{E}, \mathrm{R}$ & $\mathrm{B}, \mathrm{C}, \mathrm{L}$ & $\mathrm{O} / \mathrm{P}$ \\
\hline E. spirogyra Ehr. var. fusca Klebs & 19 & $\mathrm{E}, \mathrm{R}$ & $\mathrm{B}, \mathrm{L}$ & $\mathrm{O} / \mathrm{P}$ \\
\hline E. splendens Dang. var. splendens & 20 & $\mathrm{E}, \mathrm{R}$ & $\mathrm{A}, \mathrm{B}, \mathrm{L}$ & $\mathrm{O} / \mathrm{P}$ \\
\hline E. tripteris (Duj.) Klebs var. tripteris & 21 & $\mathrm{E}, \mathrm{R}$ & $\mathrm{B}, \mathrm{L}$ & $\mathrm{O} / \mathrm{P}$ \\
\hline E. viridis Ehr. var. viridis & 22 & $\mathrm{E}, \mathrm{R}$ & $\mathrm{B}, \mathrm{L}$ & $\mathrm{O} / \mathrm{P}$ \\
\hline Lepocinclis boseensis Xie, Qiu \& Ling & $23-25$ & $\mathrm{E}, \mathrm{R}$ & $\mathrm{B}, \mathrm{C}$ & $\mathrm{O} / \mathrm{P}$ \\
\hline L. fusiformis (Carter) Lemm. emend. Conr. var. fusiformis & 27 & $\mathrm{E}, \mathrm{R}$ & $\mathrm{B}, \mathrm{C}, \mathrm{L}$ & $\mathrm{O} / \mathrm{P}$ \\
\hline L. fusiformis (Carter) Lemm. emend. Conr. var. amphirhynchus Nyg. & 26 & $\mathrm{E}$ & B & $\mathrm{O}$ \\
\hline L. ovum (Ehr.) Lemm. var. ovum & 28 & $\mathrm{E}, \mathrm{R}$ & $\mathrm{B}, \mathrm{C}$ & $\mathrm{O} / \mathrm{P}$ \\
\hline L. ovum (Ehr.) Lemm. var. dimidio-minor Defl. & $29-30$ & $\mathrm{E}, \mathrm{R}$ & $\mathrm{B}, \mathrm{L}$ & $\mathrm{O}$ \\
\hline L. ovum (Ehr.) Lemm. var. globula (Perty) Lemm. & $31-32$ & $\mathrm{E}, \mathrm{R}$ & $\mathrm{B}, \mathrm{L}$ & $\mathrm{O} / \mathrm{P}$ \\
\hline L. playfairiana Defl. var. playfairiana & 33 & $\mathrm{E}, \mathrm{R}$ & $\mathrm{B}, \mathrm{L}$ & $\mathrm{O} / \mathrm{P}$ \\
\hline L. playfairiana Defl. var. striata Conf. & 34 & $\mathrm{R}$ & $\mathrm{B}$ & $\mathrm{P}$ \\
\hline L. salina Fritsch var. salina & 39 & $\mathrm{E}, \mathrm{R}$ & $\mathrm{B}, \mathrm{C}, \mathrm{L}$ & $\mathrm{O} / \mathrm{P}$ \\
\hline L. turbiniformis Defl. & $35-38$ & $\mathrm{R}$ & $\mathrm{L}$ & $\mathrm{P}$ \\
\hline
\end{tabular}

Todas as amostras estão depositadas na coleção de algas do Herbário Prof. Dr. Alarich R.H. Schultz (HAS) do Museu de Ciências Naturais da Fundação Zoobotânica do Rio Grande do Sul sob os números HAS 104093, HAS 104095, HAS 104096, HAS 104118, HAS 104124, HAS 104127, HAS 104128, HAS 104134, HAS 104136, HAS 104150, HAS 104174, HAS 104179, HAS 104217, HAS 104220, HAS 104337, HAS 104339, HAS 1044351, HAS 104366, HAS 104369, HAS 104370, HAS 104381, HAS 104391, HAS 104401, HAS 104416, HAS 104420, HAS 104425, HAS 104427, HAS 104431 , HAS 104432, HAS 104435, HAS 104441, HAS 104445, HAS 104455, HAS 104456.

Para a identificação específica e infra-específica dos táxons foram utilizadas obras básicas como: Conrad (1935), Gojdics (1953), Huber-Pestalozzi (1955), Pringsheim (1956), Németh (1980), Starmach (1983), Tell \& Conforti (1986), Zakrýs (1986) Wolowski (1998) e Shi et al. (1999), além de trabalhos recentes sobre o grupo como Alves-da-Silva (1988), Alves-da-Silva \& Bicudo (2002), Alves-da-Silva \& Hahn, (2004), Alves-
da-Silva \& Torres (1992), Alves-da-Silva \& Torres (1994), Cecy (1990), Ferreira \& Menezes (2000), Huszar, Menezes \& Fernandes (1989), Jati \& Train (1994), Menezes (1989, 1990) e Xavier (1989; 1994), entre outros.

\section{Resultados}

Dentre as 41 amostras analisadas, 34 apresentaram representantes de Euglenophyceae pigmentadas dos gêneros Euglena Ehrenberg e Lepocinclis Perty, permitindo a identificação de 28 táxons em nível específico e infra-específico destes dois gêneros, distribuídos em 21 variedades típicas e sete variedades que não as típicas da espécie.

\section{EUGLENACEAE}

Euglena Ehrenberg 1830.

1. Euglena acus Ehr. var. acus, Infusions. Organismen., 112, pl. 7, fig. 15. 1838.

Fig. 2 
Célula fusiforme, 88-127 $\mu \mathrm{m}$ compr., 7-9,5 $\mu \mathrm{m}$ larg., $\mathrm{Rc} / \mathrm{l}=10-13,4$.

Material examinado: HAS 104093, HAS 104095, HAS 104096, HAS 104118, HAS 104128, HAS 104134, HAS 104136, HAS 104179, HAS 104217, HAS 104337, HAS 104339, HAS 104369, HAS 104370, HAS 104391, HAS 104435.

Distribuição geográfica: cosmopolita.

2. Euglena acus Ehr. var. longissima Defl., Revue algol., 1(3): 238, pl. 4, fig. 1-3. 1924.

Fig. 3

Célula fusiforme, 230-260 $\mu \mathrm{m}$ compr., 10-13 $\mu \mathrm{m}$ larg., Rc/l = 17,8-23.

Material examinado: HAS 104093, HAS104095.

Distribuição geográfica: cosmopolita.

Difere da variedade típica pelas maiores dimensões celulares.

3. Euglena agilis Carter var. agilis, Annals. Mag. nat. Hist., 18(105): 240, pl. 6, fig. 62. 1856.

Fig. 4-5

Célula fusiforme, 20-25 $\mu \mathrm{m}$ compr., 6,5-10 $\mu \mathrm{m}$ larg., $\mathrm{Rc} / 1=3-3,8$; pólo posterior gradativamente atenuado em processo caudal hialino, cônico, ca. de $2 \mu \mathrm{m}$ compr.; película flexível, hialina, estrias finas, helicoidais; cloroplastos 2, forma de escudo, parietais, atingindo quase todo comprimento da célula, duplos-pirenóides presentes; grãos de paramido pequenos, alongados; núcleo posterior, elíptico: flagelo ca. de 0,5 vez o comprimento celular.

Material examinado: HAS 104093, HAS 104096, HAS 104337, HAS 104391, HAS 104441, HAS 104445.

Distribuição geográfica: cosmopolita.

Esta espécie apresenta deslocamento rápido, em diversas direções, mostrando acentuada metabolia com abaulamento na região anterior da célula (Fig. 5). A presença de dois cloroplastos em forma de escudo e localização parietal na célula, que atingem quase todo comprimento da célula e os duplopirenóides permitem a identificação do táxon.

4. Euglena allorgei Defl. var. allorgei, Bull. Soc. Bot. Fr., 24:116, fig. 1-2. 1924b.

Fig. 6

Célula fusiforme, 103-108 $\mu \mathrm{m}$ compr., 14-15 $\mu \mathrm{m}$ larg., $\mathrm{Rc} / \mathrm{l}=6,8-7,2$; pólo posterior gradativamente atenuado, terminando abruptamente em processo caudal hialino, cônico, voltado para um dos lados, $13 \mu \mathrm{m}$ compr.; película com estrias longitudinais; cloroplastos numerosos, alongados, discóides, parietais, $4 \mu \mathrm{m}$ compr.; grãos de paramido 2, bastoniformes, um anterior e o outro posterior ao núcleo; flagelo não observado.

Material examinado: HAS 104093, HAS104118, HAS 104136, HAS 104337, HAS 104339.

Distribuição geográfica: Europa, América do Norte, América do Sul.

Euglena allorgei Defl. é semelhante, quanto à forma da célula, a E. oxyuris Schmarda, diferindo por possuir película com estrias longitudinais e pólo posterior terminado em uma projeção voltada para um dos lados, enquanto que E. oxyuris apresenta película mais metabólica, com estrias helicoidais, que acompanham a torção da célula e pólo posterior atenuado abruptamente em processo caudal cônico, hialino, reto ou levemente inclinado.

5. Euglena caudata Hübner var. caudata, Progr., Realg. Stralsund, p. 13. 1886.

\section{Fig. 7}

Célula fusiforme, 91-105 $\mu \mathrm{m}$ compr., 18-21 $\mu \mathrm{m}$ larg., $\mathrm{Rc} / \mathrm{l}=5,0$; pólo posterior atenuado em processo caudal com ca. de $6 \mu \mathrm{m}$ compr.; película com estrias helicoidais; cloroplastos discóides, numerosos, com contorno irregular e margens lobadas, presença de duplopirenóides; grãos de paramido numerosos, pequenos, alongados; núcleo central a posterior; flagelo de 0,5 a 1 vez o comprimento celular.

Material examinado: HAS 104179, HAS 104391, HAS 104445.

Distribuição geográfica: América do Norte, América do Sul, Ásia, Europa.

6. Euglena deses Ehr. var. deses Klebs. Unters. d. bot. Inst. Tübingen, 1(2): 303. 1883.

Fig. 8

Célula cilíndrica a oblonga, 92,5-101,7 $\mu \mathrm{m}$ compr., 9,2-10,2 $\mu \mathrm{m}$ larg., Rc/1 = 9,9-11; pólo posterior abruptamente atenuado em processo caudal hialino, obtuso, mamilado; película semi-rígida, estrias tênues, helicoidais; cloroplastos numerosos, discóides, parietais; pirenóides nus; grãos de paramido numerosos, bastoniformes; núcleo elíptico, central; flagelo 0,1-0,2 vez o comprimento da célula.

Material examinado: HAS 104134, HAS 104136, HAS 104179, HAS 104337, HAS 104370, HAS 104441, HAS 104445.

Distribuição geográfica: cosmopolita.

7. Euglena ehrenbergii Klebs var. ehrenbergii, Unters. d. bot. Inst. Tübingen, 1: 304,1883.

Fig. 9 
Célula cilíndrica, 88-302 $\mu$ m compr., 9-30 $\mu$ m larg., $\mathrm{Rc} / \mathrm{l}=6,2-9,7$.

Material examinado: HAS 104118, HAS 104134, HAS 104337, HAS 104369, HAS 104370, HAS 104391, HAS 104401, HAS 104416.

Distribuição geográfica: cosmopolita.

8. Euglena limnophila Lemm. var. limnophila, Beih. Bot. Zbl., 76(44-45): 152. 1898.

Fig. 10

Célula fusiforme, 76-88,2 $\mu \mathrm{m}$ compr., 10-15,2 $\mu \mathrm{m}$ larg., $\mathrm{Rc} / \mathrm{l}=6,8-7,2$.

Material examinado: HAS 104093, HAS 104095 , HAS 104337, HAS 104391.

Distribuição geográfica: cosmopolita.

Esta variedade é morfologicamente semelhante a E. gaumei All. \& Lefr., sendo que E. gaumei se apresenta mais metabólica, com menores dimensões celulares, o processo caudal ligeiramente inclinado e as estrias longitudinais, enquanto que em E. limnophila var. limnophila, as estrias são espiraladas ou tão tênues sendo muito difícil sua visualização.

9. Euglena limnophila Lemm. var. minor Drez., Kosmos, 50: 245, 268. 1925.

Fig. 11

Célula fusiforme, 50-60 $\mu \mathrm{m}$ compr., 10-12 $\mu \mathrm{m}$ larg., $\mathrm{Rc} / 1=4-5$.

Material examinado: HAS 104093, HAS 104095. Distribuição geográfica: América do Sul, Europa.

Difere da variedade típica pelas menores dimensões celulares e menor relação comprimento/ largura ( $\mathrm{Rc} / \mathrm{l})$.

10. Euglena mutabilis Schmitz var. mutabilis, Jb. wiss. Bot., 15: 37, pl. 1, fig. 3, 1884.

Fig. 12

Célula estreitamente fusiforme a cilíndrica, 95-128 $\mu$ m compr., 8-13 $\mu \mathrm{m}$ larg., Rc/l = 9,8-10.

Material examinado: HAS 104093, HAS 104118, HAS 104134, HAS 104174, HAS 104336, HAS 104401, HAS 104425, HAS 104435, HAS 104441.

Distribuição geográfica: América do Norte, América do Sul, Ásia, Europa.

A variedade apresenta movimento reptante intenso, o que dificulta sua identificação. Sladecéck (1973), entre outros autores, menciona que a espécie é encontrada em ambientes ácidos. No Rio Grande do Sul este táxon tem mostrado ampla tolerância a esta variável ambiental, ocorrendo em ambientes desde ácido até básico, mas com maior incidência em águas ácidas (Alves-da-Silva \& Torres 1994; Alves-da-Silva \& Hahn 2004).

11. Euglena oxyuris Schmarda var. oxyuris, Beitr. Nat. Infus. 17, pl. 1, fig. 17. 1846.

Fig. 13

Célula cilíndrica, 194-260 $\mu \mathrm{m}$ compr., 22-25 $\mu \mathrm{m}$ larg.; Rc/l = 7,6-11,4.

Material examinado: HAS 104095, HAS 104118, HAS 104127, HAS 104150, HAS 104217, HAS 104431, HAS 104337, HAS 104339, HAS 104427.

Distribuição geográfica: cosmopolita.

12. Euglena polymorpha Dang. var. polymorpha, Le Botaniste, 8: 175, fig. 12. 1901.

Fig. 14-15

Célula fusiforme 80-90 $\mu$ m compr., 15-19 $\mu$ m larg., $\mathrm{Rc} / \mathrm{l}=4,2-4,7$; pólo anterior arredondado; pólo posterior atenuado gradativamente em processo caudal cônico; película com estrias helicoidais; cloroplastos numerosos, discóides, com margens irregulares; duplo-pirenóides presentes; grãos de paramido numerosos, discóides; núcleo central a posterior; flagelo ca. de 0,5 vez o comprimento da célula.

Material examinado: HAS 104381.

Distribuição geográfica: América do Norte, América do Sul, Ásia, Europa.

13. Euglena sanguinea Ehr. var. sanguinea, Abh. Berl. Akad. Wiss. Physik aus d. Jahre 1831, Berlin, p. 7.1832.

Fig. 16-17

Célula amplamente fusiforme a fusiforme, 93-110 $\mu \mathrm{m}$ compr., 25-32 $\mu \mathrm{m}$ larg., Rc/1 = 2,9-4,4.

Material examinado: HAS 104118, HAS 104337, HAS 104369, HAS 104427, HAS 104431.

Distribuição geográfica: cosmopolita.

Euglena sanguinea tem sido citada por vários autores como uma espécie que quando em floração, pode conferir coloração avermelhada a água, devido à presença de grânulos de hematocromo na célula da alga. No presente estudo foram registrados poucos indivíduos por lâmina, não tendo sido observada floração, nem coloração das águas.

A presença de numerosos grânulos de hemacromo distribuídos em toda a célula ou mais concentrados na região central, dificulta a observação dos cloroplastos e pirenóides, sendo somente possível visualizar estas duas estruturas, em espécimens que apresentam poucos grânulos de hematocromo, tal como ocorreu 

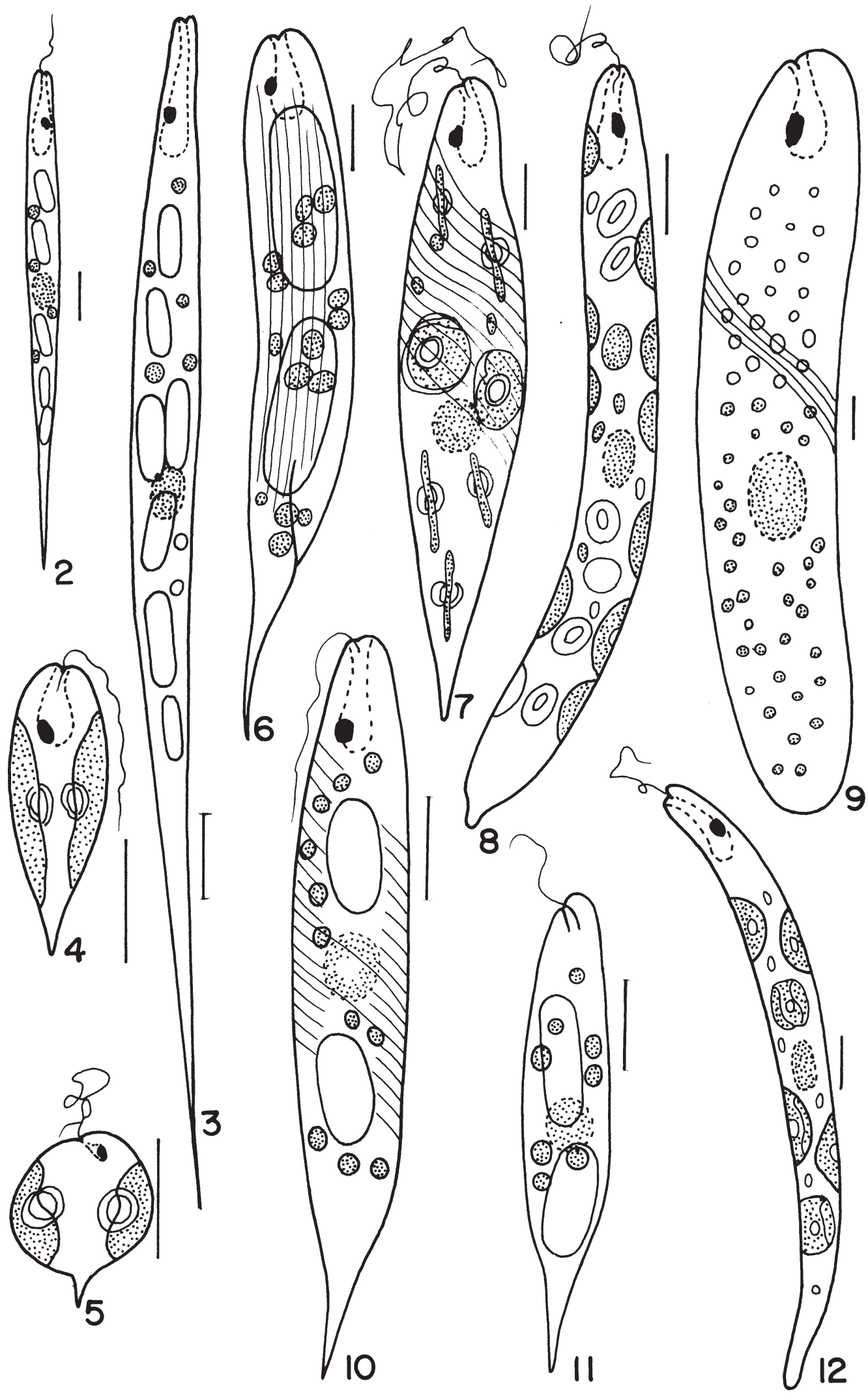

Figuras 2-12. 2. Euglena acus Ehr. var. acus. 3. Euglena acus Ehr. var. longissima Defl. 4-5. Euglena agilis Carter var. agilis. 5. Célula em metabolia. 6. Euglena allorgei Defl. var. allorgei. 7. Euglena caudata Hübner var. caudata. 8. Euglena deses Ehr. var. deses Klebs 9. Euglena ehrenbergii Klebs var. ehrenbergii. 10. Euglena limnophila Lemm. var. limnophila. 11. Euglena limnophila Lemm. var. minor Drez. 12. Euglena mutabilis Schmitz var. mutabilis. Escala $=10 \mu \mathrm{m}$. 
em alguns indivíduos observados.

14. Euglena spirogyra Ehr. var. spirogyra, Abh. Berl. Akad. Wiss. Physik aus d. Jahre 1930, p. 83. 1932.

Fig. 18

Célula cilíndrica, 80-88 $\mu$ m compr., 10-15 $\mu$ m larg., $\mathrm{Rc} / \mathrm{l}=7,3-8$.

Material examinado: HAS 104093, HAS 104096, HAS 104124, HAS 104134, HAS 104337, HAS 104366, HAS 104391, HAS 104455.

Distribuição geográfica: cosmopolita.

Este táxon é facilmente identificado pela presença de grânulos na película. Os indivíduos ora registrados apresentaram ampla variabilidade quanto a coloração e a ornamentação da película como já havia sido citado por Alves-da-Silva \& Hahn (2004) em material coletado no Parque Estadual Delta do Jacuí.

15. Euglena spirogyra Ehr. var. fusca Klebs, Unters. bot. Instit. Tübingen, 1: 77. 1883.

Fig. 19

Célula fusiforme 139-148 $\mu \mathrm{m}$ compr., 23-28 $\mu \mathrm{m}$ larg., $\mathrm{Rc} / \mathrm{l}=5,0-5,9$.

Material examinado: HAS 104093, HAS 104096, HAS 104118, HAS 104128, HAS 104179, HAS 104337 , HAS 104369, HAS 104401, HAS 104416, HAS 104435.

Distribuição geográfica: cosmopolita.

Esta variedade difere da típica pelas maiores dimensões celulares, pelos grânulos poligonais e coloração da película castanha-avermelhada.

16. Euglena splendens Dang. var. splendens, Le Botaniste, 8: 69, fig. 9. 1901.

Fig. 20

Célula fusiforme, 75-83 $\mu$ m compr.,17-22 $\mu \mathrm{m}$ larg., $\mathrm{Rc} / \mathrm{l}=3,8-4,2$.

Material examinado: HAS 104118, HAS 104370, HAS 104427, HAS 104431, HAS 104432.

Distribuição geográfica: cosmopolita.

17. Euglena tripteris (Duj.) Klebs var. tripteris, Unters. bot. Inst. Tübingen, 1: 306. 1883.

Fig. 21

Célula fusiforme, 78-82 $\mu \mathrm{m}$ compr., 10-15,5 $\mu \mathrm{m}$ larg., Rc/l = 5,3-7,8.

Material examinado: HAS 104093, HAS 104095 , HAS 104118, HAS 104127, HAS 104134, HAS 104136, HAS 104174, HAS 104179, HAS 104337, HAS 104339.
Distribuição geográfica: cosmopolita.

Esta variedade é morfologicamente semelhante a E. oxyuris diferindo desta pela maior torção celular da célula, pelo corte óptico transversal trirradiado e processo caudal levemente inclinado.

18. E. viridis Ehr. var. viridis, Infus. 107, pl. 7, fig. 9. 1838.

Fig. 22

Célula fusiforme, 44-50 $\mu \mathrm{m}$ compr., 10-14 $\mu \mathrm{m}$ larg., Rc/1 =3,6-4,4; pólo anterior arredondado; pólo posterior atenuado em processo caudal cônico; película com estrias finas, helicoidais; cloroplastos numerosos em fitas radiais, partindo da região central da célula orientadas em diversas direções; grãos de paramido numerosos, arredondados, concentrados na região central da célula; núcleo posterior, arredondado, flagelo ca. de 1 vez o comprimento celular.

Material examinado: HAS 104093, HAS 104174, HAS 104179, HAS 104432, HAS 104435, HAS 104445.

Distribuição geográfica: cosmopolita.

Lepocinclis Perty 1852.

19. Lepocinclis boseensis Xie, Qiu \& Ling, Acta Phytotax. Sin. 34: 226, fig. 1: 1. 1996.

Fig. 23-25

Célula elíptica, 41-43 $\mu$ m compr., 18-22 $\mu$ m larg., $\mathrm{Rc} / \mathrm{l}=1,9-2,3$; pólo anterior aquadradado, atenua-se levemente terminando em um pólo truncado, abertura do canal apical; pólo posterior levemente atenuado em processo caudal, reto, de 6-9 $\mu$ m compr.; película com estrias levógiras; cloroplastos numerosos, discóides, ca. de 3,0 $\mu \mathrm{m}$ diâm.; grãos de paramido 1-2, anelares, laterais, opostos ou centrais; flagelo ca. de 0,5 vez o comprimento celular.

Material examinado: HAS 104093, HAS 104337, HAS 104391.

Distribuição geográfica: Ásia.

Esta espécie é morfologicamente semelhante a Lepocinclis ovum var. ovum, diferenciando-se pelo pólo anterior mais quadrático e truncado. É o primeiro registro da espécie para as Américas.

20. Lepocinclis fusiformis (Carter) Lemm. emend. Conr. var. fusiformis, Arch. Protistenk., 82(2): 225, fig. 30. 1934.

Fig. 26

Célula citriforme a elíptica, 34-36 $\mu \mathrm{m}$ compr., $23 \mu \mathrm{m}$ larg., $\mathrm{Rc} / \mathrm{l}=1,5-1,6$. 


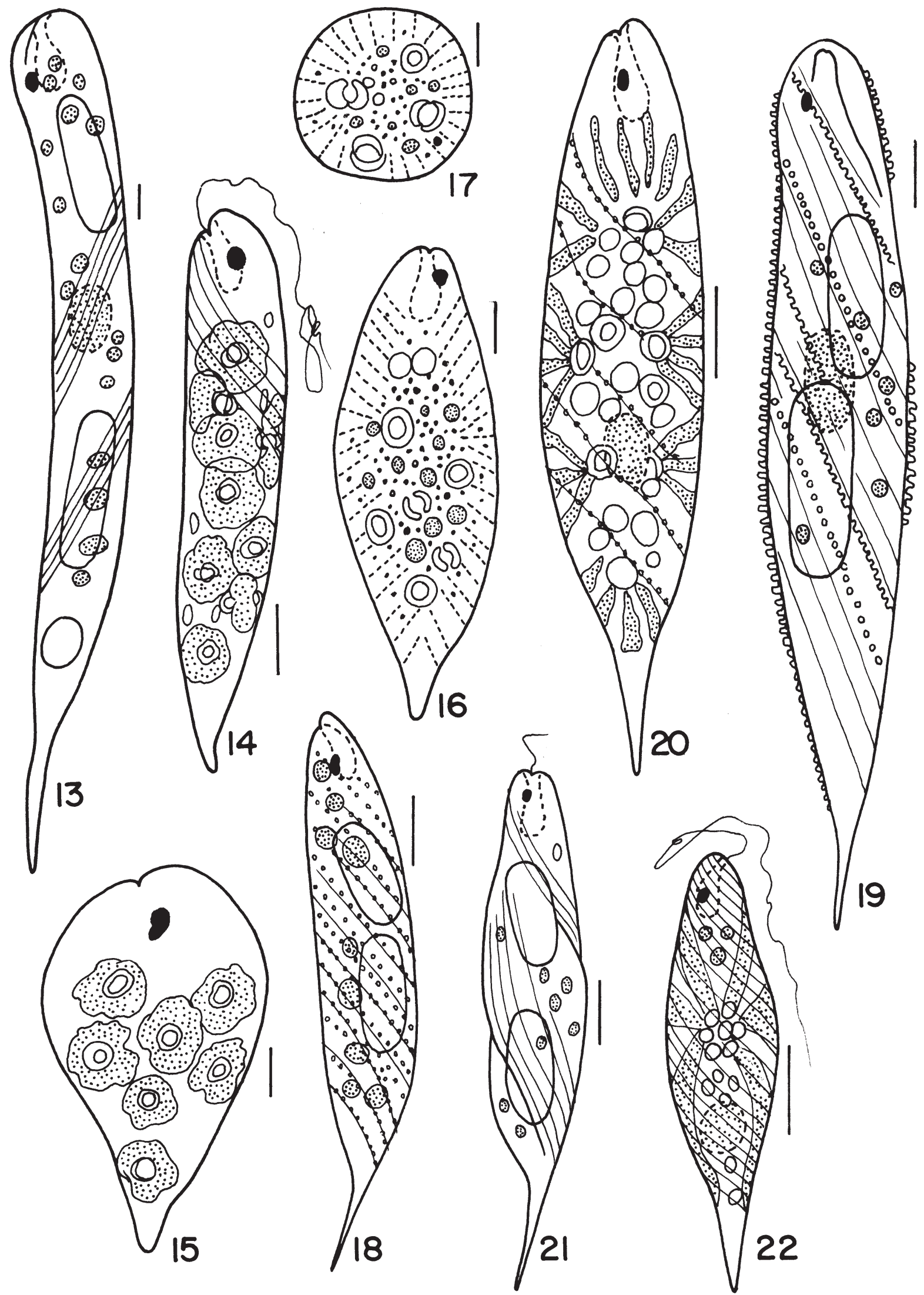

Figuras 13-22. 13. Euglena oxyuris Schmarda var. oxyuris. 14-15. Euglena polymorpha Dang. var. polymorpha 16-17. Euglena sanguinea Ehr. var. sanguinea. 17. Vista polar. 18. Euglena spirogyra Ehr. var. spirogyra. 19. Euglena spirogyra Ehr. var. fusca Klebs. 20. Euglena splendens Dang. var. splendens. 21. Euglena tripteris (Duj.) Klebs var. tripteris. 22. E. viridis Ehr. var. viridis. Escala $=10 \mu \mathrm{m}$. 
Material examinado: HAS 104093, HAS 104118, HAS 104217, HAS 104369, HAS 104391, HAS 104416.

Distribuição geográfica: cosmopolita.

21. Lepocinclis fusiformis (Carter) Lemm. emend. Conr. var. amphirhynchus Nyg., Dank. Vid. Selsk. Biol. Skr., 7(1): 167, fig. 101. 1949.

Fig. 27

Célula citriforme, ca. de 31,5 $\mu$ m compr., ca. de $25 \mu \mathrm{m}$ larg., $\mathrm{Rc} / \mathrm{l}=1,3$; pólo anterior com mamilo obtuso proeminente ca. de $4 \mu \mathrm{m}$ de compr. por $5 \mu \mathrm{m}$ de largura, pólo posterior levemente atenuado terminando em mamilo; película com estrias levógiras; cloroplastos numerosos, discóides, de 2,0-2,5 $\mu$ m diâm.; grãos de paramido 2, anelares, laterais, opostos; núcleo e flagelo não observados.

Material examinado: HAS104124.

Distribuição geográfica: África, América do Sul, Ásia.

A variedade diferencia-se da típica pelo pólo anterior mais proeminente.

22. Lepocinclis ovum (Ehr.) Lemm. var. ovum, Kryptogamenfl. Bradenburg, 3: 504, fig. 13. 1910.

Fig. 28

Célula elíptica, 27,7-30 $\mu \mathrm{m}$ compr., 17,6-20,8 $\mu \mathrm{m}$ larg., $\mathrm{Rc} / \mathrm{l}=1,4-1,7$.

Material examinado: HAS 104093, HAS 104096, HAS 104118, HAS 104369, HAS 104391.

Distribuição geográfica: cosmopolita.

23. Lepocinclis ovum (Ehr.) Lemm. var. dimidiominor Defl., Bull. Soc. bot. Fr., 24(4): 1121, fig. 25-28. 1924.

Fig. 29-30

Célula estreitamente-elíptica a elíptica, 16,2-18,5 $\mu \mathrm{m}$ compr., 9,2-12 $\mu \mathrm{m}$ larg., Rc/l = 1,4-1,9.

Material examinado: HAS 104093, HAS 104095 , HAS 104118, HAS 104128, HAS 104134, HAS 104136, HAS 104174, HAS 104179, HAS 104217, HAS 104339, HAS 104369, HAS 104370, HAS 104391, HAS 104445.

Distribuição geográfica: cosmopolita.

Difere da variedade típica pelas menores dimensões celulares.

24. Lepocinclis ovum (Ehr.) Lemm. var. globula (Perty) Lemm., Kryptogamenfl. Bradenburg, 3(4): 505. 1910.

Fig. 31-32

Célula subglobosa, 19-20 $\mu \mathrm{m}$ compr., 15-18 $\mu \mathrm{m}$ larg., $\mathrm{Rc} / \mathrm{l}=1,1-1,2$; pólo posterior levemente atenuado terminando em mamilo de $0,5 \mu \mathrm{m}$ de compr.; película com estrias levógiras; cloroplastos numerosos, discóides, de 1,5 $\mu \mathrm{m}$ diâm.; grãos de paramido 2, anelares, laterais, opostos; núcleo e flagelo não observados.

Material examinado: HAS 104124, HAS 104136, HAS 104366, HAS 104369.

Distribuição geográfica: cosmopolita.

Difere da espécie típica pela forma da célula mais larga. Alguns exemplares encontrados no Pontal do Anastásio, subárea G, na primavera de 2003 apresentaram maiores dimensões que as citadas por Conrad (1935) para esta forma, coincidindo com as dimensões citadas por Tell \& Conforti (1986) para Argentina e Alves-da-Silva \& Bicudo (2002) no Estado do Rio Grande do Sul, Brasil.

25. Lepocinclis playfairiana Defl. var. playfairiana, Ann. de Protist., 2: 16, fig. 23. 1929.

Fig. 33

Célula amplamente fusiforme ou elíptica, ca. de $46 \mu \mathrm{m}$ compr., ca. de $23 \mu \mathrm{m}$ larg., $\mathrm{Rc} / \mathrm{l}=2$; pólo anterior assimétrico, com uma saliência lembrando bico; pólo posterior levemente atenuado em processo caudal, reto ou ligeiramente curvo, ca. de $10 \mu \mathrm{m}$ compr., película lisa; cloroplastos numerosos, discóides, ca. de 3,0 $\mu \mathrm{m}$ diâm.; grãos de paramido 1-2, anelares, laterais, opostos ou centrais; flagelo ca. de 0,5 vez o comprimento celular.

Material examinado: HAS 104337.

Distribuição geográfica: cosmopolita.

26. Lepocinclis playfairiana Defl. var. striata Conf., Nova Hedwigia 53(1-2): 79, pl.2, fig. 9. 1991.

Fig. 34

Célula amplamente fusiforme, 40-42 $\mu \mathrm{m}$ compr., 20-22 $\mu \mathrm{m}$ larg., $\mathrm{Rc} / \mathrm{l}=1$,9-2,0; pólo anterior assimétrico, com uma saliência lembrando bico; pólo posterior levemente atenuado em processo caudal, reto, de 6-9 $\mu \mathrm{m}$ compr.; película com estrias levógiras; cloroplastos numerosos, discóides, ca. de 3,0 $\mu$ m diâm.; grãos de paramido 1-2, anelares, laterais, opostos ou centrais; flagelo ca. de 0,5 vez o comprimento celular.

Material examinado: HAS 104337.

Distribuição geográfica: América do Sul.

Difere da variedade típica pela presença de estrias levógiras e pelo processo caudal mais reto. Espécie só encontrada na América do Sul.

27. Lepocinclis salina Fritsch var. salina, New Phytologist, 13: 351, fig. 3a-b, e. 1914.

Fig. 35 
Célula amplamente ovada a ovada, $42-52 \mu \mathrm{m}$ compr., 30,5-45 $\mu \mathrm{m}$ larg., Rc/1 = 1,1-1,6.

Material examinado: HAS 104093, HAS 104118, HAS 104136, HAS 104220, HAS 104337, HAS 104369, HAS 104391, HAS 104396, HAS 104401, HAS 104416, HAS 104420, HAS 104425, HAS 104445, HAS 104456.

Distribuição geográfica: cosmopolita.

Este táxon é morfologicamente semelhante a L. texta var. texta (Duj.) Lemm. sendo a diferença diagnóstica mais marcante o sentido das estrias que na var. salina são dextrógiras, enquanto na var. texta são levógiras.

28. Lepocinclis turbiniformis Defl., Bull. Mus. Hist. nat. de Paris, 6: 422. 1926.

Fig. 36-39

Célula turbiniforme, 36-45 $\mu \mathrm{m}$ compr., 20-25 $\mu \mathrm{m}$ larg., $\mathrm{Rc} / \mathrm{l}=1,8$; corte óptico transversal circular; pólo anterior arredondado; abertura do canal apical, pólo posterior atenuado abruptamente em processo caudal cônico, reto, de 10-11 $\mu \mathrm{m}$ compr.; película rígida, hialina; estrias levógiras; cloroplastos numerosos, parietais, discóides, ca. de $3 \mu \mathrm{m}$ diâm.; grãos de paramido 2, anelares, laterais; flagelo não observado.

Material examinado: HAS 104369.

Distribuição geográfica: América do Sul e Europa.

Esta espécie é morfologicamente muito semelhante a Lepocinclis piriformis Cunha distinguindo-se pela maior largura, dimensões celulares, e pelo sentido das estrias que em L. turbiniformis são levógiras, enquanto $L$. piriformis são dextrógiras. Os espécimens ora observados, distinguiram-se da descrição do autor da espécie, pelas maiores dimensões celulares e maior processo caudal.

Wetzel (1993) citou que as euglenofíceas são mais encontradas em águas pouco profundas e ricas em matéria orgânica. Muitos dos ambientes estudados são pequenos corpos d'água rasos cobertos por macrófitas aquáticas que ao se decomporem favorecem o aumento de matéria orgânica, principalmente nas margens onde foi realizada a maioria das coletas analisadas. Dentre estes locais se sobressaiu em especial, um banhado entre as Lagoas do Capivari e do Casamento, na subárea A, com a presença de 14 táxons de Euglena e quatro de Lepocinclis.

Do total das 34 amostras em que houve registro destes dois gêneros, Lepociclis salina var. salina foi a espécie que ocorreu em maior número de amostras analisadas (41,2\%). Outras espécies que se distinguiram mas em menor porcentagem foram
Euglena acus var. acus, E. spirogyra var. fusca, E. tripteris, Lepocinclis ovum var. dimidio-minor, sendo registradas em menos de $38 \%$ do total das amostras analisadas.

A maioria dos táxons foi encontrada nas duas estações do ano, com exceção de Euglena acus var. longissima, E. limnophila var. minor, Lepocinclis fusiformis var. amphirhynchus e L. ovum var. dimidio-minor registrados somente no outono e Euglena polymorpha var. polymorpha, Lepocinclis playfairiana var. striata e L. turbiniformis só encontrados na primavera (Tab. 1).

A maioria dos táxons identificados $(78,6 \%)$ ocorre tanto no fitoplâncton quanto no perifíton (Tab. 1).

Na primavera de 2003, os banhados associados à Lagoa do Casamento apresentaram maior riqueza específica dos dois gêneros. Embora tenha sido registrada uma alta riqueza nestes ambientes, observouse que a maioria dos táxons de Euglena e Lepocinclis apresentaram poucos indivíduos por lâmina.

Euglena acus var. acus, E. acus var. longissima, E. oxyuris var. charkowiensis, E. oxyuris var. oxyuris, E. spirogyra, E. spirogyra var. fusca, Lepocinclis fusiformis var. fusiformis e L. ovum var. dimidio-minor já haviam sido registrados no litoral sul, na área da Estação Ecológica do Taim por Alves-daSilva (1988).

O gênero Euglena foi o que apresentou o maior número de táxons (18). Dentre estes, dez são cosmopolitas e oito tem distribuição restrita a menos de quatro continentes. Dos dez táxons do gênero Lepocinclis, quatro têm distribuição restrita, enquanto os demais são cosmopolitas.

Lepocinclis playfairiana var. striata é exclusivo da América do Sul e L. boseensis até a presente data, estava restrito à China, sendo esta a primeira citação para as Américas e a segunda em nível mundial.

São novos registros para o estado do Rio Grande do Sul e país, L. playfairiana var. playfairiana e L. boseensis.

\section{Agradecimentos}

Ao Conselho Nacional de Desenvolvimento Científico e Tecnológico (CNPq), pela concessão de Bolsa de Iniciação Científica (PIBIC) à segunda autora (proc. 108097/03-1); aos colegas da Seção de Botânica de Criptógamas do Museu de Ciências Naturais da Fundação Zoobotânica do Rio Grande do Sul, pela execução e auxílio nas coletas; a Rejane Rosa, pela cobertura à nanquim dos desenhos; a Eduardo da Silva 

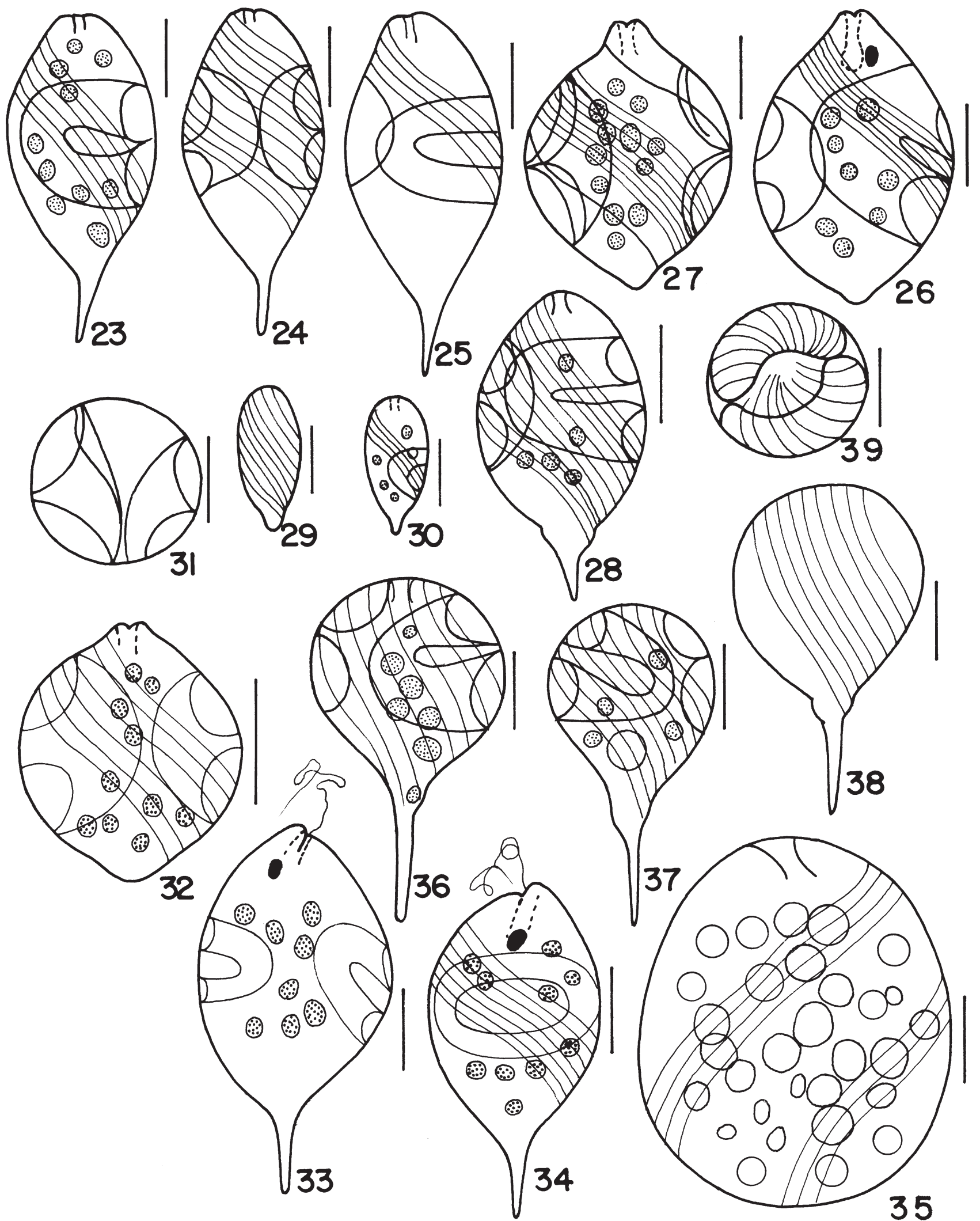

Figuras 23-39. 23-25. Lepocinclis boseensis Xie, Qiu \& Ling. 26. Lepocinclis fusiformis (Carter) Lemm. emend. Conr. var. fusiformis. 27. Lepocinclis fusiformis (Carter) Lemm. emend. Conr. var. amphirhynchus Nyg. 28. Lepocinclis ovum (Ehr.) Lemm. var. ovum. 29-30. Lepocinclis ovum (Ehr.) Lemm. var. dimidio-minor Defl. 31-32. Lepocinclis ovum (Ehr.) Lemm. var. globula (Perty) Lemm. 31. Vista polar. 33. Lepocinclis playfairiana Defl. var. playfairiana. 34. Lepocinclis playfairiana Defl. var. striata Conf. 35. Lepocinclis salina Fritsch var. salina. 36-39. Lepocinclis turbiniformis Defl. 39. Vista polar. Escala $=10 \mu \mathrm{m}$. 
Pinheiro do Laboratório de Geoprocessamento, pela elaboração do mapa.

\section{Referências bibliográficas}

Alves-da-Silva, S.M. 1988. Euglenaceae pigmentadas (Euglenophyta) da Estação Ecológica do Taim, Rio Grande do Sul, Brasil. Iheringia (38): 109-126.

Alves-da-Silva, S.M. \& Bicudo, C.E.M. 2002. Contribution to the knowledge of genus Lepocinclis (Euglenophyceae) of the state of Rio Grande do Sul, Southern Brazil. Algological Studies 106: 77-97.

Alves-da-Silva, S.M. \& Hahn, A.T. 2004. Study of Euglenophyta in the Jacuí Delta State Park, Rio Grande do Sul, Brazil. 1. Euglena Ehr., Lepocinclis Perty. Acta Botanica Brasilica 18(1): 123-140.

Alves-da-Silva, S.M. \&. Torres, J.R. 1992. Estudo taxonômico do gênero Lepocinclis Perty (Família Euglenaceae), Parque Zoológico de Sapucaia do Sul e Jardim Botânico de Porto Alegre, Rio Grande do Sul, Brasil. Iheringia 42: 87-104.

Alves-da-Silva, S.M. \& Torres, J.R. 1994. O gênero Euglena Ehr. de sistemas lênticos do Parque Zoológico e Jardim Botânico, Rio Grande do Sul, Brasil. Revista Brasileira de Biologia 54(2): 345-363.

Cecy, I.I.T. 1990. A restinga do Pontal do Sul, município de Paranaguá, Pr. - I. Levantamento ficológico (Euglenophyta) e físico-químico. Arquivos de biologia e tecnologia 33(1): 1-79.

Conrad, W. 1935. Etude systématique du genre Lepocinclis Perty. Musée Royal Naturelle de Belgique 1: 1-85.

Ferreira, A.C.S. \& Menezes, M. 2000. Flora planctônica de um reservatório eutrófico, lagoa Guandu, município de Nova Iguaçu, RJ. Hoehnea 27(1): 45-76.

Gojdisc, M. 1953. The genus Euglena. The Madison, The University of Wisconsin Press.

Huber-Pestalozzi, G. 1955. Euglenophyceen. Pp. 1-605. In: G. Huber-Pestalozzi (ed.). Das phytoplankton des Susswässers: Systematik und Biologie. Teil 4, E. Schweizerbart'sche Verlangsbuchhandlug, Stuttgart.

Huszar, V.L.M.; Menezes, M. \& Fernandes, V.O. 1989. Fitoplâncton de rede da lagoa Cabiúnas, Macaé, estado do Rio de Janeiro, Brasil: uma contribuição a seu conhecimento. Boletim do Museu Nacional 78: $1-43$.
Jati, S. \& Train, S. 1994. Euglenaceae pigmentadas de ambientes lênticos da ilha Porto Rico, município de Porto Rico, Paraná, Brasil. Iheringia (45): 117-142.

Menezes, M. 1989. Contribuição ao conhecimento de algas pigmentadas do gênero Euglena (Euglenophyceae) no município do Rio de Janeiro e arredores, Brasil. Acta Botanica Brasilica 3(1): 49-90.

Menezes, M. 1990. Estudos taxonômicos sobre o gênero Lepocinclis Perty ( Euglenaceae) no Município do Rio de Janeiro e arredores, Brasil. Revista Brasileira de Biologia 50(1): 103-113.

Németh, J. 1980. Az ostoros Algák (Euglenophyta). Budapeste, Vizdock (Hidrobiologie Séries n. 8), vol. 1.

Pringsheim, E.G. 1956. Contributions towards a monograph of the genus Euglena. Nova Acta Leopoldense 18(125): $1-168$.

Shi, Z.; Wang, Q.; Xie, S.; Dai, J. \& Chen, L. 1999. Euglenophyta. (s.l.) Science Press. Flora Algarum Sinicarum Aquae Dulcis, t. 6.

Sladecék, V. 1973. System of water quality from the biological point of view. Archiv für Hydrobiologie 7: 1-218.

Starmach, K. 1983. Euglenophyta. Pp. 1-593. In: K. Starmach (ed.). Flora Slodkowodna Polski. Polska Academia Nauk., Warszawa, v. 3.

Tell, G. \& Conforti, V.T.D. 1986. Euglenophyta pigmentadas de la Argentina. Berlim Stuttgart, J. Cramer.

Wetzel, R.G. 1993. Limnologia. 2 ed. Lisboa, Fundação Carlouste Gulbenkian.

Wolowski, K. 1998. Taxonomic and environmental studies on euglenophytes of the Kraków-Czestochowa Upland (Southern Poland). Fragmenta Floristica et Geobotanica, Suppl. 6: 3-192.

Xavier, M.B. 1989. O gênero Lepocinclis Perty de lagos do Parque Estadual das Fontes do Ipiranga, São Paulo, Brasil. Hoehnea 16: 133-147.

Xavier, M.B. 1994. Criptógamos do Parque Estadual das fontes do Ipiranga, São Paulo, SP. Algas, 5: Euglenophyceae (Euglenaceae pigmentadas). Hoehnea 2(1-2): 47-73.

Zakrýs, B. 1986. Contribution to the monograph of polish members of the Genus Euglena Ehr. Nova Hedwigia 42(2/4): 491-540.

Zakrýs, B.; Cambra-Sanchez, J. \& Walne, P. 2001. Chloroplasts ultrastruture of Euglena cuneata Pringsheim, E. deses Ehrenberg and E. mutabilis (Euglenophyceae): taxonomy significance. Acta Protozoologica 40: 161-167. 